\title{
AN EXTREMAL PROPERTY OF SIMPLICES
}

\author{
G. J. BUTLER
}

ABSTRACT. It is shown that the class of $n$-dimensional simplices is an extremal for the problem of maximizing the volume of the minimum circumscribing cylinder to an $n$-dimensional convex body of unit volume.

1. It is a simple consequence of a result of Macbeath (Lemma 6 of [1]) that every compact convex body $K$ in $E^{n}$ is contained in a cylinder whose volume is no greater than $n$ times that of $K$. By a cylinder, we shall mean any affine equivalent of the cartesian product of an $(n-1)$-dimensional convex set and a line-segment.

The purpose of this note is to show that the class of $n$-dimensional simplices is an extremal for the problem of maximizing the volume of the circumscribing cylinder to a convex body. This will follow from the result quoted above and the following

THEOREM. Every circumscribing cylinder to an n-dimensional simplex has volume at least $n$ times that of the simplex.

We note that a standard approximation argument using the Blaschke selection theorem (see, for example, [4]) shows that for any compact convex body $K$ in $E^{n}$, the value

$$
\underset{C \supset k}{\inf } \mu_{n}(C), \quad C \text { a cylinder, }
$$

where $\mu_{n}(\cdot)$ denotes $n$-dimensional measure, is attained for some cylinder $C^{\prime} \supset K$.

We introduce some notation. Let $x=\left(\xi_{1}, \cdots, \xi_{n}\right)$ be a general point of $E^{n}$. We shall denote the hyperplane $\xi_{n}=0$ by $P_{0}$, and the parallel plane $\xi_{n}=1$ by $P_{1}$. If $A \subset E^{n}$ is a compact set, we use conv $A$ to denote its convex hull, and if $a$ is a vector in $E^{n}$ which is not parallel to $P_{0}$, we shall use $\pi(a, A)$ to denote the shadow of $A$ on $P_{0}$ in the direction $\boldsymbol{a}$, that is

$$
\pi(\mathbf{a}, A)=\{\mathrm{x}+\mathrm{ta} \mid \mathrm{x} \in A,-\infty<t<\infty\} \cap P_{0} .
$$

If $S$ is an $n$-dimensional simplex, we define $V(S)$ to be its vertex set and $V_{i}(S)$ to be $V(S) \cap P_{i}, i=0,1$.

Received by the editors October 12, 1970.

AMS 1969 subject classifications. Primary 5240.

Key words and phrases. Simplex, circumscribing cylinder, shadow.

Copyright (c) 1971, American Mathematical Society 
2. In this section we shall prove a number of lemmas that we shall use in proving the Theorem. Before stating these, however, we remark that since the problem is invariant under affine transformations, we need to concern ourselves only with those simplices $S$ for which $P_{0}$ and $P_{1}$ are tac-planes (we shall denote the family of such simplices by $\delta$ ) and with those cylinders whose bases are shadows on to $P_{0}$ of $S$ in the direction a of their generators. Accordingly, the problem becomes that of minimizing

$$
f(a, S)=\mu_{n-1}[\pi(a, S)] / \mu_{n}(S),
$$

for $S \in \mathcal{S}$ and a nonparallel to $P_{0}$. The lemmas are

Lemma 1. Let $S \in \mathcal{S}$ and let a be a vector, nonparallel to $P_{0}$. Then there exists a simplex $S^{*} \in \mathcal{S}^{*}$, the family of $n$-dimensional simplices whose vertex sets are contained in $P_{0} \cup P_{1}$, for which $f\left(a, S^{*}\right) \leqq f(a, S)$.

Lemma 2. Let $S \in \mathcal{S}^{*}$ and suppose that $V_{0}(S) \cap \pi\left(a, V_{1}(S)\right) \neq \varnothing$. Then $f(a, S)=n$.

Lemma 3. Let $S \in \mathcal{S}^{*}$ and let a be a vector nonparallel to $P_{0}$. Suppose, further, that $\operatorname{conv}\left(V_{0}(S)\right) \cap \operatorname{conv}\left[\pi\left(a, V_{1}(S)\right)\right] \neq \varnothing$. Then $f(a, S) \geqq n$.

Lemma 4. Let $X_{1}, X_{2}$ be subsets of $E^{n}$. Then conv $X_{1}+$ conv $X_{2}$ $=\operatorname{conv}\left(X_{1}+X_{2}\right)$.

Proof of Lemma 1. Let $V(S)=\left\{\mathrm{x}_{0}, \mathrm{x}_{1}, \cdots, \mathrm{x}_{n}\right\}$. If $S \in \mathcal{S}^{*}$, there is nothing to prove. Suppose, then, that one of the vertices, say $\mathbf{x}_{0}$, lies strictly between $P_{0}$ and $P_{1}$. Then there exist numbers $t_{0}, t_{1}$ of opposite sign, for which

$$
\mathrm{x}_{0}+t_{i} \mathrm{a} \in P_{i}, \quad i=0,1 .
$$

Let $\boldsymbol{b}$ be a unit vector orthonormal to the hyperplane containing $\mathbf{x}_{1}, \mathbf{x}_{2}, \cdots, \mathbf{x}_{n}$, and let $T$ denote $\operatorname{conv}\left(\bigcup_{i=0}^{n} x_{i}\right)$. Then for any real number $t$, we have

$$
\begin{aligned}
\mu_{n}\left[\operatorname{conv}\left\{x_{0}+a, x_{1}, \cdots, x_{n}\right\}\right] & =\frac{1}{n}\left|\left(x_{0}+t a\right) \cdot b\right| \mu_{n-1}(T) \\
& =\left|\mu_{n}(S)+\frac{t}{n}(a \cdot b)\right| \mu_{n-1}(T) .
\end{aligned}
$$

It follows that one of the two simplices

$$
S_{i}=\operatorname{conv}\left\{\mathrm{x}_{0}+t_{i} \mathbf{a}, \mathrm{x}_{1}, \cdots, \mathrm{x}_{n}\right\}, \quad i=0,1,
$$

satisfies the inequalities 


$$
\mu_{n}\left(S_{i}\right) \geqq \mu_{n}(S), \quad \mu_{n-1}\left[\pi\left(a, S_{i}\right)\right]=\mu_{n-1}[\pi(a, S)],
$$

and

$$
\operatorname{card}\left(V_{0}\left(S_{i}\right) \cup V_{1}\left(S_{i}\right)\right)>\operatorname{card}\left(V_{0}(S) \cup V_{1}(S)\right)
$$

Repeating this argument as many times as necessary, we obtain a simplex with the desired properties.

Proof of Lemma 2. Without loss of generality, suppose that $\mathbf{x}_{0}, \mathbf{x}_{1}, \cdots, \mathbf{x}_{h} \in P_{0}: \mathbf{x}_{h+1}, \mathbf{x}_{h+2}, \cdots, \mathbf{x}_{n} \in P_{1}$, where $V(S)=\left\{\mathbf{x}_{0}, \mathbf{x}_{1}, \cdots, \mathbf{x}_{n}\right\}$, and $0 \leqq h \leqq n-1$, and that $\pi\left(a, x_{n}\right)=x_{0}$.

Then we can find an affine transformation of $E^{n}$ that takes $x_{0}$ into $\mathbf{0}, \mathbf{x}_{\boldsymbol{i}}$ into $\boldsymbol{e}_{i}, i=1, \cdots, h, \mathbf{x}_{i}$ into $\boldsymbol{e}_{i}+\boldsymbol{e}_{n}, i=h+1, \cdots, n-1$, and $x_{n}$ and $a$ into $e_{n}$, where $e_{1}=(1,0, \cdots, 0,0), e_{2}=(0,1, \cdots, 0,0), \cdots$, $e_{n}=(0,0, \cdots, 0,1)$.

Then under this transformation it is immediate that $\mu_{n}\left(S_{n}\right)=1 / n$ !, $\mu_{n-1}\left[\pi\left(a, S_{n}\right)\right]=1 /(n-1)$ !, and the result follows.

Proof of Lemma 3. Assume, as in Lemma 2, that $\bigcup_{i=0}^{h} x_{i} \subset P_{0}$, $\bigcup_{i=h+1}^{n} x_{i} \subset P_{1}$, with $0 \leqq h \leqq n-1$, where $V(S)=\left\{x_{0}, x_{1}, \cdots, x_{n}\right\}$. Suppose that $c \in \operatorname{conv} V_{0}(S) \cap \operatorname{conv}\left[\pi\left(a, V_{1}(S)\right)\right]$ and let $d \in \operatorname{conv} V_{1}(S)$ such that $\pi(\boldsymbol{a}, \boldsymbol{b})=\mathbf{c}$. Define $S_{\boldsymbol{i}}$ to be conv $\left[\boldsymbol{d} \cup V(S) \sim \boldsymbol{x}_{\boldsymbol{i}}\right], i=h+1$, $\cdots, n$, and $A_{i}$ to be $\pi\left(a, S_{i}\right)$. Then the $S_{i}$ are (possibly degenerate) simplices and the $A_{i}$ are their shadows onto $P_{0}$ in the direction $a$. We assert that

(a) $\bigcup_{i=n+1}^{n} S_{i}=S$;

(b) $\bigcup_{i=h+1}^{n} A_{i}=\pi(a, S)$;

(c) $\mu_{n}\left(S_{i} \cap S_{j}\right)=0$ for $i \neq j$;

(d) $\mu_{n-1}\left(A_{i} \cap A_{j}\right)=0$ for $i \neq j$.

We omit the proof which is an elementary application of vector algebra. From (a)-(d) we deduce the existence of an $n$-dimensional simplex $S_{m} \in \mathcal{S}^{*}$ where $h+1 \leqq m \leqq n$ such that $f\left(\mathbf{a}, S_{m}\right) \leqq f(a, S)$, and conv $V_{0}\left(S_{m}\right) \cap \pi\left[a, V_{1}\left(S_{m}\right)\right] \neq \varnothing$.

Now we repeat the above argument with $S_{m}$ in place of $S$ and with the roles of $P_{0}$ and $P_{1}$ interchanged to obtain a simplex $\bar{S} \in \mathcal{S}^{*}$ which satisfies the conditions of Lemma 2 and for which $f(a, \bar{S}) \leqq f(a, S)$. Lemma 3 now follows.

Proof of Lemma 4. Let $x \in \operatorname{conv}\left(X_{1}+X_{2}\right)$. Then $x=\lambda\left(a_{1}+a_{2}\right)$ $+(1-\lambda)\left(b_{1}+b_{2}\right)$, where $0 \leqq \lambda \leqq 1, a_{i} \in X_{i}, b_{i} \in X_{i}, i=1,2$, and so $x=\left(\lambda a_{1}+(1-\lambda) b_{1}\right)+\left(\lambda a_{2}+(1-\lambda) b_{2}\right) \in \operatorname{conv} X_{1}+\operatorname{conv} X_{2}$. Thus $\operatorname{conv}\left(X_{1}+X_{2}\right) \subset$ conv $X_{1}+$ conv $X_{2}$. On the other hand suppose $y \in$ conv $X_{1}+$ conv $X_{2}$. Then $y=\lambda_{1} a_{1}+\left(1-\lambda_{1}\right) b_{1}+\lambda_{2} a_{2}+\left(1-\lambda_{2}\right) b_{2}$, where $0 \leqq \lambda_{i} \leqq 1, a_{i} \in X_{i}, b_{i} \in X_{i}, i=1,2$. Without loss of generality, assume that $\lambda_{1} \leqq \lambda_{2}$. Then $y=\lambda_{1}\left(a_{1}+a_{2}\right)+\left(\lambda_{2}-\lambda_{1}\right)\left(b_{1}+a_{2}\right)$ 
$+\left(1-\lambda_{2}\right)\left(b_{1}+b_{2}\right) \in \operatorname{conv}\left(X_{1}+X_{2}\right)$. Thus $\operatorname{conv}\left(X_{1}+X_{2}\right) \subset$ conv $X_{1}$ $+\operatorname{conv} X_{2}$ and the lemma is proved.

3. We are now ready to prove the theorem. After Lemma 1, we need only consider simplices in $\delta^{*}$. Let $S \in \delta^{*}$, a be any vector nonparallel to $P_{0}$. Let $V(S)=\left\{\mathbf{x}_{0}, \mathbf{x}_{1}, \cdots, \mathbf{x}_{n}\right\}$ and assume that

$$
\bigcup_{i=0}^{h} \mathbf{x}_{i} \subset P_{0}, \quad \bigcup_{i=h+1}^{n} \mathbf{x}_{i} \subset P_{1}, \quad \text { where } 0 \leqq h \leqq n-1 .
$$

Let $\mathbf{x}_{i}^{\prime}$ be $\pi\left(e_{n}, \mathbf{x}_{i}\right), i=0,1, \cdots, n$. Define $D$ to be the set

$$
\left\{u \mid u \in P_{0} \text { and conv } V_{0}(S) \cap \operatorname{conv}\left[\pi\left(e_{n}+u, V_{1}(S)\right)\right] \neq \varnothing\right\} \text {. }
$$

We shall show that

(1) $D$ is the $(n-1)$-dimensional convex hull $H$ of the set of vectors

$$
y_{i j}=x_{i}^{\prime}-x_{j}^{\prime}, \quad i=0,1, \cdots, h ; j=h+1, \cdots, n .
$$

In fact, we can show that $D$ is a convex polytope with $(h+1)(n-h)$ vertices, but this fact will not be needed and so the proof is omitted. Clearly $D \supset H$. Let $\mathrm{x} \in D$. Then there is a vector $y \in \operatorname{conv} V_{0}(S)$ $\cap \operatorname{conv}\left[\pi\left(e_{n}+x, V_{1}(S)\right)\right]$, and so $y=c=d+x$, where $c \in \operatorname{conv} V_{0}(S)$, and $d \in \operatorname{conv} \pi\left(e_{n}, V_{1}(S)\right)$, and so $x \in \operatorname{conv} V_{0}(S)-\operatorname{conv} \pi\left(e_{n}, V_{1}(S)\right)$. By Lemma $4, x \in \operatorname{conv}\left[V_{0}(S)-\pi\left(e_{n}, V_{1}(S)\right)\right]=H$. Thus $D=H$.

Now the set $W$ of vectors

$$
\mathbf{x}_{0}^{\prime}-\mathbf{x}_{h+1}^{\prime}, \mathbf{x}_{1}^{\prime}-\mathbf{x}_{h+1}^{\prime}, \cdots, \mathbf{x}_{h}^{\prime}-\mathbf{x}_{h+1}^{\prime}, \mathbf{x}_{h}^{\prime}-\mathbf{x}_{h+2}^{\prime}, \cdots, \mathbf{x}_{h}^{\prime}-\mathbf{x}_{n}^{\prime}
$$

is an (n-1)-dimensional set. For the dimension of $W$ is that of the space spanned by the set

$$
\begin{aligned}
x_{1}^{\prime}-x_{0}^{\prime}, x_{2}^{\prime}-x_{0}^{\prime}, \cdots, x_{h}^{\prime}-x_{0}^{\prime}, \\
\\
\left(x_{h+1}^{\prime}-x_{h+2}^{\prime}+x_{h}^{\prime}-x_{0}^{\prime}\right), \cdots,\left(x_{h+1}^{\prime}-x_{n}^{\prime}+x_{h}^{\prime}-x_{0}^{\prime}\right),
\end{aligned}
$$

which is that of the space spanned by the set

$$
\mathbf{x}_{1}-\mathbf{x}_{0}, \mathbf{x}_{2}-\mathbf{x}_{0}, \cdots, \mathbf{x}_{h}-\mathbf{x}_{0}, \mathbf{x}_{h+1}-\mathbf{x}_{h+2}, \cdots, \mathbf{x}_{h+1}-\mathbf{x}_{n},
$$

which is at most one less than that of the space spanned by the set

$$
\mathbf{x}_{1}-\mathrm{x}_{0}, \mathbf{x}_{2}-\mathbf{x}_{0}, \cdots, \mathbf{x}_{h}-\mathbf{x}_{0}, \mathbf{x}_{h+1}-\mathbf{x}_{0}, \mathbf{x}_{h+2}-\mathbf{x}_{0}, \cdots, \mathbf{x}_{h}-\mathbf{x}_{0},
$$

which is $n$.

Now $W \subset D$, and this finishes the proof of (1). Next we introduce the function

$$
g(u)=\mu_{n-1}\left[\pi\left(e_{n}+u, S\right)\right]=\mu_{n}(S) f\left(e_{n}+u, S\right),
$$


where $u$ is a variable vector in $P_{0}$. By [3], this is a convex function of $u$. By (1), Lemmas 2 and 3, and the convexity of $g$, it follows that $g(\boldsymbol{u})=n \mu_{n}(S)$ on $D$. By (1), $D$ has nonempty interior in $P_{0}$ and so, again using the convexity of $g$, it follows that

$$
g(u) \geqq n \mu_{n}(S), \quad u \in P_{0} .
$$

Thus $f(a, S) \geqq n$, which proves the theorem.

4. Remarks. 1. It is possible to show that, in fact,

$$
f(a, S)>n \quad \text { whenever } \pi\left(e_{n}, a\right) \in P_{0} \sim D .
$$

2. Rogers and Shephard [2] define a convex body $K$ to be a pseudodouble pyramid if it is possible to find points $a, b$ on the boundary of $K$ such that each 2-dimensional section of $K$ by a plane through $a$ and $b$ is either a triangle with $a$ and $b$ as vertices, or a convex quadrilateral with $a$ and $b$ as opposite vertices. If we call $a-b$ the direction of the axis of such a body, it follows from [2] and the Theorem that for every direction $\xi$, a simplex is a pseudodouble pyramid with axis in that direction.

3. I believe that the simplex is the only class of extremals for the maximization problem discussed, but have been unable to prove this.

ACKNowledgment. This work represents part of the author's Ph.D. thesis submitted to the University of London in 1968. The author wishes to record his gratitude to Professor C. A. Rogers for his valuable assistance as supervisor, and to the Science Research Council for their financial support.

\section{REFERENCES}

1. A. M. Macbeath, $A$ compactness theorem for affine equivalence-classes of convex regions, Canad. J. Math. 3 (1951), 54-61. MR 13, 577.

2. C. A. Rogers and G. C. Shephard, Some extremal problems for convex bodies, Mathematika 5 (1958), 93-102. MR 21 \#2960.

3. G. C. Shephard, Shadow systems of convex sets, Israel J. Math. 2 (1964), 229236. MR 31 \#3931.

4. F. A. Valentine, Convex sets, McGraw-Hill Series in Higher Math., McGrawHill, New York, 1964. MR $30 \# 503$.

University of Alberta, Edmonton, Alberta, Canada 